\title{
MODELLING THE DUST EMISSION OF THE L1551 IRS5 BINARY SYSTEM
}

\author{
M. Osorio, ${ }^{1}$ P. D'Alessio, ${ }^{2}$ J. Muzerolle, ${ }^{3}$ N. Calvet, ${ }^{4}$ and L. Hartmann ${ }^{4}$ \\ RESUMEN
}

Modelamos la fuente L1551 IRS5 como un sistema de dos protoestrellas con sus discos circunestelares, los cuales a su vez están rodeados por un disco circunbinario. Todos los elementos del sistema todavía están inmersos su envoltura natal, que se encuentra en colapso. Con este modelo calculamos autoconsistentemente la distribución espectral de energía de la fuente, y reproducimos las absorciones características de agua y silicato, así como la distribución espacial de brillo de la envolvente y de los discos. Comparando el modelo con las observaciones determinamos los parametros de todas las componentes del sistema. Encontramos que una envoltura achatada es la más adecuada para explicar las observaciones y que la tasa de acreción de material en esta envoltura es mucho mayor que la tasa de accreción en los discos circunestelares.

\section{ABSTRACT}

We model the L1551 IRS5 source as a system containing two protostars, each surrounded by a circumstellar disk, both encircled by a circumbinary disk, and all disks surrounded by an extended infalling flattened envelope. With this composite model, we can calculate self-consistently the spectral energy distribution of the source, the shape of the ice and silicate features, and the spatial intensity distributions of the envelope and disks. We compare our model results with the observations, determining the physical parameters of the disks and the envelope. We find that flattened envelope collapse models are required in order to explain the observations, and that the infall rate of the envelope is much larger than the accretion rates of the binary disks.

Key Words: BINARIES: GENERAL — STARS: CIRCUMSTELLAR MATTER - STARS: FORMATION - STARS: INDIVIDUAL (L1551 IRS5)

\section{INTRODUCTION}

L1551 IRS5 is a prototypical Class I source. It is located in a nearby star-forming region $(d=140 \mathrm{pc})$, and it is the first object where the molecular bipolar outflow phenomenon was discovered. For these reasons, it has been one of the most studied young stellar objects (YSOs), and a wide database is available. At present, it is known that L1551 IRS5 is a binary system of two protostars, separated by 45 $\mathrm{AU}$, each of them surrounded by a circumstellar disk (CS) of radius $\sim 10 \mathrm{AU}$; both CS disks are encircled by a much larger circumbinary disk (CB), which is still surrounded by a dense and large envelope of radius $\sim 8000$ AU (see left panel of Fig. 1). In this paper we present a model of the dust emission of L1551 IRS5, including all these components. From the results of this modelling we infer the properties of each component of the system and we determine their contribution to the emission observed with different instruments and wavelengths.

\footnotetext{
${ }^{1}$ Instituto de Astrofísica de Andalucía, CSIC, Spain. email: osorio@iaa.es

${ }^{2}$ Centro de Radioastronomía y Astrofísica, UNAM, Mexico.

${ }^{3}$ Steward Observatory, University of Arizona, USA.

${ }^{4}$ Harvard-Smitsonian Center for Astrophysics, USA.
}

\section{MODELLING AND RESULTS}

We calculate the emergent flux of the envelope using the radiative transfer methods developed by Calvet et al. (1994), applied to envelope models with different degrees of asphericity and inclinations. For the CS disks, we assume flared disks heated by viscous dissipation and by the irradiation of the envelope. Since the CB disk is large, its main source of heating is the irradiation from the envelope, and its surface density and temperature can be represented by power laws. In the calculations of $\mathrm{CS}$ and $\mathrm{CB}$ disks we adopt the procedures and approximations described in D'Alessio, Calvet \& Hartmann (1997). In the following, we describe the properties obtained for the different components.

The properties of the envelope are mostly determined by the single-dish total flux density and spatial intensity distribution in the $\mathrm{mm}$ wavelength range (see references in Osorio et al 2003). A very flattened envelope with an inclination angle similar to that of the CS disks, and an infall accretion rate of $\sim 7 \times 10^{-5} M_{\odot} \mathrm{yr}^{-1}$ is required to explain the observations. We find that the emission of this enevlope is the dominant component in the 3 to $200 \mu \mathrm{m}$ wavelength range covered by the $I S O$ data points (White 

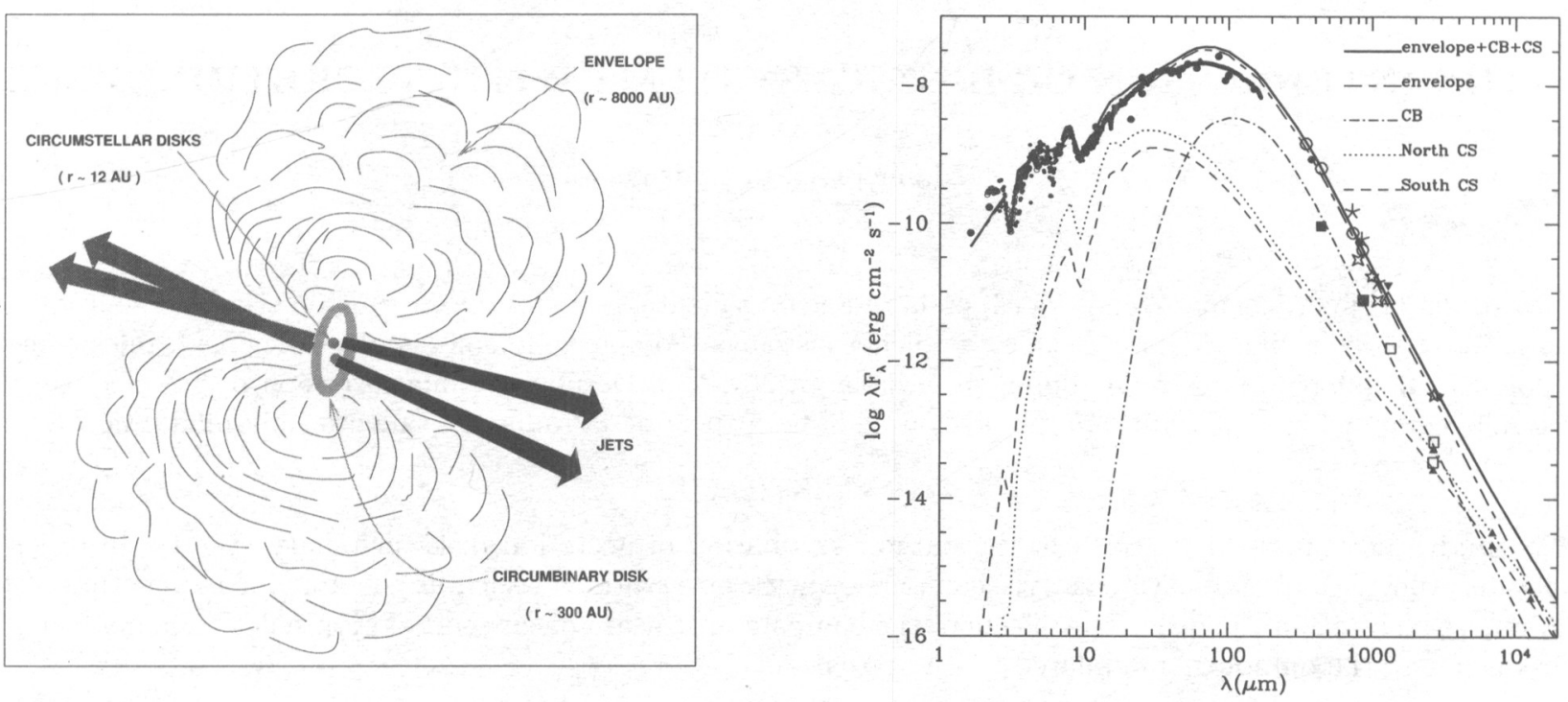

Fig. 1. (Left) Sketch of L1551 IRS5, showing the individual components. (Right) Observed data points (see references in Osorio et al. 2003), and model predicted SEDs for the L1551 IRS5 system.

et al. 2000; see Fig. 1, right panel).

The properties of the CS disks are inferred mainly from high-angular resolution interferometric data in the $\mathrm{mm}$ range, that resolve out the emission of the envelope (see Osorio et al 2003 and references therein). We note that for the set of parameters obtained, the emission of the CS disks is optically thick, even in the mm range, which is in contrast with what is generally assumed. Furthermore, we find that the CS disks are inclined in such a way that their outer parts hide the emission coming from their innermost regions. Their emission peaks at mid-infrared wavelengths $(\sim 30 \mu \mathrm{m})$, but is considerably lower than the ISO values (Fig. 1, right panel).

The properties of the CB disk are not well constrained by the data, but its contribution, in addition to that of the envelope, is required to explain the observed sub-mm flux densities. The best fit is obtained for a $\mathrm{CB}$ disk of radius $\sim 300 \mathrm{AU}$ and mass ranging from $0.02-0.4 M_{\odot}$. Its emission dominates that of the CS disks in the range $\sim 60-2000 \mu \mathrm{m}$, even though it is lower than that of the envelope.
From our fit, we obtain a value of $25 L_{\odot}$ for the total luminosity of the system, and $\sim 55^{\circ}$ for the inclination angle. The mass infall rate of the envelope is an order of magnitude, or more, larger than the total accretion rate through the two CS disks. This might suggest an accumulation of material in the $\mathrm{CB}$ disk, possibly with occasional cascades of accretion into the binary system.

M.O. acknowledges support from MCYT grant AYA 2002-00376 (including FEDER funds) and from Junta de Andalucía (Spain).

\section{REFERENCES}

Calvet, N., Hartmann, L., Kenyon, S. J., \& Whitney, B. A. 1994, ApJ, 434, 330

D'Alessio, P., Calvet, N., \& Hartmann, L. 1997, ApJ, 474,397

Osorio, M., D'Alessio, P., Muzerolle, J., Calvet, N., \& Hartmann, L. 2003, ApJ, 586, 1148

White, G. J. et al. 2000, A\&A, 364, 741 